
allemande

48-2 | 2016

Les espaces publics des pays germanophones, des espaces publics transnationaux?

\title{
Das Ende der Nachkriegszeit? Die Teilnahme Deutschlands an den Gedenkfeierlichkeiten zum D- Day 2004 und 2014
}

Jean-Samuel Marx

\section{OpenEdition}

\section{Journals}

Édition électronique

URL : https://journals.openedition.org/allemagne/424

DOI : 10.4000/allemagne.424

ISSN : 2605-7913

Éditeur

Société d'études allemandes

Édition imprimée

Date de publication : 28 décembre 2016

Pagination : $317-328$

ISSN : 0035-0974

Référence électronique

Jean-Samuel Marx „Das Ende der Nachkriegszeit? Die Teilnahme Deutschlands an den Gedenkfeierlichkeiten zum D-Day 2004 und 2014", Revue d'Allemagne et des pays de langue allemande

[Online], 48-2 | 2016, Online erschienen am: 28 Dezember 2017, abgerufen am 18 Mai 2021. URL: http://journals.openedition.org/allemagne/424 ; DOI: https://doi.org/10.4000/allemagne.424 


\title{
Das Ende der Nachkriegszeit? Die Teilnahme Deutschlands an den Gedenkfeierlichkeiten zum D-Day 2004 und 2014
}

\author{
- Jean-Samuel Marx*
}

Am 6. Juni 2004 nahm Gerhard Schröder als erster deutscher Bundeskanzler an den Gedenkveranstaltungen an die Landung der Alliierten in der Normandie im Jahre 1944 Teil. Der Ablauf der Feierlichkeiten macht die große Bedeutung deutlich, die dieser Premiere beigemessen wurde. Neben der internationalen Eröffnungszeremonie in Arromanches und den jeweils nationalen Gedenkfeiern wurden unterschiedliche bilaterale Gedenkfeiern zwischen Frankreich und den wichtigsten an der Landung beteiligten Staaten organisiert. Von großer symbolischer Bedeutung ist deshalb die Tatsache, dass die Feierlichkeiten mit einer gemeinsamen Zeremonie mit dem alten „Erbfeind“ Deutschland abgeschlossen wurden. Als Ort wurde ein Geschichtsmuseum über den Zweiten Weltkrieg, der „Mémorial de la Paix“ in Caen, gewählt. Jacques Chirac und Gerhard Schröder wurden von der deutsch-französischen Brigade unter Beifall der Anwesenden empfangen, enthüllten daraufhin eine Gedenktafel und hielten schließlich eine Rede, die mit einer Umarmung und einem sogenannten „Bad in der Menge“ der anwesenden französischen und deutschen Gymnasiasten endete ${ }^{(1)}$. Dass Deutschland erstmals dazu eingeladen wurde, zusammen mit den ehemaligen Siegern des Kriegs des Anfangs des Endes der Nazi-Herrschaft zu gedenken, ist ein Zeichen dafür, dass sich etwas geändert hat. Steht diese hoch symbolische Geste aber für das endgültige Ende der Nachkriegszeit, wie Gerhard Schröder im Vorfeld der Gedenkfeier erklärte? Gibt es inzwischen so etwas wie eine „deutsche Normalität“? Wie präsentiert sich die Bundesrepublik sechzig Jahre nach Ende des Zweiten Weltkriegs und fünfzehn Jahre nach dem Mauerfall in Frankreich und wie wird sie dort wahrgenommen? Diesen Fragen soll im Folgenden nachgegangen werden. Interessant ist schließlich auch die

* Doctorant, Universités de Heidelberg et de Strasbourg, EA 1341 Études germaniques.

1 Ein ausführlicher Bericht befindet sich zum Beispiel in: Marie-Estelle PECH, „France-Allemagne, l'émotion“, Le Figaro, 07.06.2004. 
Frage, ob das gemeinsame Gedenken Frankreichs und Deutschlands an den D-Day mit einer Überwindung der jeweils nationalen Erinnerungskultur einherging, was wiederum die Frage aufwirft, ob nun von einer gemeinsamen, deutsch-französischen Öffentlichkeit die Rede sein kann. Angesichts des besonderen Kontextes des Jahres 2004, auf den noch eingegangen werden soll, erscheint es sinnvoll, in einem letzten Schritt einen Blick auf das Jahr 2014 zu werfen: Kann der 6. Juni 2014 als eine Bestätigung des 6. Juni 2004 betrachtet werden oder waren die Feierlichkeiten zum 60. Jahrestag des D-Day ein einmaliges Ereignis, das in erster Linie dem nationalen und internationalen Kontext geschuldet war? Die Untersuchung erfolgte anhand von Reden, Interviews und Gastbeiträgen der Staatsvertreter Deutschlands und Frankreichs sowie von Pressekommentaren aus den Zeitungen Le Monde, Le Figaro, Libération, La Croix und L'Humanité, womit die überregionalen Tageszeitungen gewählt wurden, die die höchste Auflage haben und möglichst das politische Spektrum abbilden.

Dass die Anwesenheit Gerhard Schröders in der Normandie eine so große symbolische Bedeutung besitzt, ist darauf zurückzuführen, dass Deutschland mit dieser Geste symbolisch in den „Kreis der Siegermächte“ aufgenommen wurde, und dass die alten „Erbfeinde“ Deutschland und Frankreich, die sich in zahlreichen Kriegen bekämpft haben, nun gemeinsam der Verbrechen des Zweiten Weltkriegs gedachten. „Vor fünfzig Jahren war es undenkbar. Vor zehn Jahren kam es nicht dazu. Am Sonntag wird zum ersten Mal ein deutscher Kanzler an den Gedenkveranstaltungen der Landung in der Normandie teilnehmen“(2). So beginnt ein Artikel der Journalistin Odile Benyahia-Kouider in Libération am 5. Juni 2004. Doch wie lässt sich diese Entwicklung erklären? Warum ist 2004 die Anwesenheit eines deutschen Staatsvertreters möglich geworden? Natürlich darf der Faktor Zeit nicht unterschätzt werden: Es waren immerhin sechzig Jahre seit dem Kriegsende vergangen und die Generation, die im Zweiten Weltkrieg gekämpft, bzw. ihn erlebt hat, starb langsam ab. Auch in dieser Hinsicht kam den Feierlichkeiten eine besondere Bedeutung zu: Es war den Organisatoren bewusst, dass beim nächsten großen Jubiläum nur noch eine Handvoll Veteranen würde dabei sein können. Doch es wäre verkürzt, die Einladung Deutschlands allein auf den wachsenden zeitlichen Abstand zum Krieg zu reduzieren.

Fest steht zunächst, dass das Deutschland, das Gerhard Schröder in der Normandie vertritt, nicht dasselbe Land ist wie nach 1945 und auch nicht das gleiche wie unmittelbar nach der Wiedervereinigung. Gerhard Schröder legte im Vorfeld und während der Gedenkfeier viel Wert darauf, dies hervorzuheben. In seiner Rede am 6. Juni 2004 in Caen erklärte er: „Es ist nicht das alte Deutschland jener finsteren Jahre, das ich hier vertrete. Mein Land hat den Weg zurück in den Kreis der zivilisierten Völkergemeinschaft gefunden. Es war ein langer Weg zu einer erfolgreichen und stabilen Demokratie“(3). Ähnliche Aussagen finden sich in einem Gastbeitrag in der Regionalzeitung Ouest France und in einem Interview mit Le Figaro im Vorfeld der Feierlichkeiten. In seinen

2 Siehe Odile Benyahia-Kouider, „Soixante ans après, l'Allemagne aux côtés des Alliés“, Libération, 05.06.2004. Im Original heißt es: „Il y a cinquante ans, ćétait impensable. Il y a dix ans, cela ne s'est pas fait. Dimanche, pour la première fois, un chancelier allemand participera aux commémorations du débarquement en Normandie.“

3 Siehe „Rede von Bundeskanzler Gerhard Schröder bei den französisch-deutschen Feierlichkeiten des ,D-Day‘ am 6. Juni 2004 in Caen“, Bulletin der Bundesregierung, Nr. 58-4 vom 06.06.2004. 
Äußerungen machte Gerhard Schröder die Bedeutung deutlich, die seiner Meinung nach dem Ende der deutschen Teilung in diesem Prozess zukommt: In Ouest France erklärte er, dass der „Mut und die Solidarität“ der DDR-Bürger erforderlich gewesen sei, „damit ein deutscher Bundeskanzler endlich wieder im Namen eines freien, demokratischen, vereinigten, souveränen Landes sprechen konnte, eines Landes, das Ver-

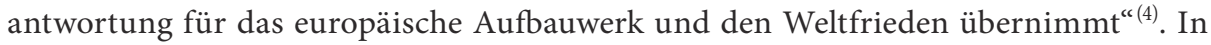
seinem langen Interview mit Le Figaro ging er näher auf den letztgenannten Aspekt ein und sprach von den internationalen Verpflichtungen, die mit der Wende 1989/90 einhergegangen seien, womit er die allmähliche Aufgabe der sogenannten ScheckbuchDiplomatie ab Mitte der $1990^{\text {er }}$ Jahre rechtfertigte ${ }^{(5)}$. Gerhard Schröder stellte sich als Vertreter eines neuen Deutschlands dar, eines Landes, das aus den Fehlern der Vergangenheit gelernt habe und von dem nun keine Gefahr mehr ausgehe. In Le Figaro ging er noch weiter und sprach von „Normalität“. Was bedeutet all dies? In seinen 2006 veröffentlichten Memoiren schrieb der Altkanzler einen interessanten Satz: Erst mit der Einladung zur Teilnahme am D-Day-Gedenken schien ihm „wirklich das Ende der Nachkriegszeit gekommen" zu sein, zumal seine Anwesenheit keine nennenswerten Proteste ausgelöst habe. Und fügte hinzu: „Der deutsche Gast war willkommen“(6). Gerhard Schröder deutete also seine Einladung durch Jacques Chirac als Beweis für das Vertrauen der Weltgemeinschaft in Deutschland und somit auch als Beleg für die Richtigkeit seiner These des „,neuen Deutschlands“. Mit der erwähnten Hervorhebung der Rolle von Mauerfall und Wiedervereinigung in diesem Wandel machte er auch deutlich, dass eine deutsche Teilnahme an den Feierlichkeiten zum D-Day vor 1990 undenkbar gewesen wäre. Erst ein vereintes und damit auch voll souveränes Land konnte nämlich durch sein Engagement für Europa und die Übernahme von mehr Verantwortung in der Welt - auch im militärischen Bereich - den Beweis für seine Veränderung erbringen. So die, zwar implizite, Argumentation Gerhard Schröders. Die Betonung darauf, dass Deutschland aus den Fehlern der Vergangenheit gelernt habe, ermöglichte ihm des Weiteren einen etwas unverkrampfteren Umgang mit eben dieser Vergangenheit. Auch das ist Ausdruck des „neuen Deutschlands“, das er verkörpern wollte. Das wird an zwei Stellen seiner Rede deutlich. Einmal, als er neben den gestorbenen alliierten Soldaten auch an die deutschen Soldaten erinnerte, die im Krieg gefallen sind, „weil sie in einen mörderischen Feldzug zur Unterdrückung Europas geschickt wurden“. Und ein anderes Mal bei der Bewertung des deutschen Widerstands. Diesbezüglich erklärte er, dass der 6. Juni "für viele Deutsche" das endgültige militärische Scheitern symbolisiert habe, dass „andere Deutsche“ aber „schon lange zuvor erkannt [hätten], dass mit der national-sozialistischen Gewaltherrschaft der moralische Untergang Deutschlands begonnen hatte". Viele von ihnen hätten es mit dem Tod bezahlt ${ }^{(7)}$. Die Botschaft beider Aussagen: Nicht alle Deutschen waren Nazis, und auch Deutsche waren Opfer. In seinem Gastbeitrag in Ouest France machte Gerhard Schröder schließlich den 6. Juni

4 Siehe „Bundeskanzler Gerhard Schröder: Namensartikel in der Zeitung Ouest France“, Presse- und Informationsamt der Bundesregierung, 05.06.2004.

5 Siehe Gerhard SchröDer, „Ces morts, nous les avons tous en commun“, Le Figaro, 05.06.2004.

6 Siehe Gerhard Schröder, Entscheidungen - Mein Leben in der Politik, Hamburg, 2006, S. 640.

7 Siehe „Rede von Bundeskanzler Gerhard Schröder“ (Anm. 3). 
1944 zum Beginn der „Befreiung Frankreichs, Deutschlands und ganz Europas vom schrecklichen Joch der Nazis“(8) und knüpfte damit an die berühmte Rede von Bundespräsident Richard von Weizsäckers (1984-1994) an, der im Jahr 1985 den 8. Mai 1945 zum „Tag der Befreiung“ erklärt hatte ${ }^{(9)}$. Solche Äußerungen wären seitens eines deutschen Kanzlers bei einem Auftritt im Ausland lange undenkbar gewesen, 2004 haben sie keine Debatte mehr ausgelöst ${ }^{(10)}$. Ein nicht unwichtiger Grund für den Auftritt dieses „neuen Deutschlands“ ist zweifellos die Tatsache, dass Gerhard Schröder, Jahrgang 1944, zu einer Generation von Politikern gehört, die für die Verbrechen der Hitler-Diktatur nicht mehr verantwortlich gemacht werden kann. Im Gegensatz etwa zu Helmut Kohl, 1930 geboren, hat er den Krieg nicht einmal mehr als Kind erlebt. Das ist ein Aspekt, der auch in französischen Pressekommentaren genannt wird ${ }^{(11)}$. Hoch interessant ist aber, dass Gerhard Schröder in seiner Rede darauf hinwies, dass er die Gräueltaten des Krieges trotzdem nie vergessen werde. Fast als Beweis dafür erzählte er seinen Zuhörern in Caen vom Schicksal seiner Familie: Seinen Vater habe er nie kennengelernt, er sei ihm Krieg gefallen und dessen Grab sei im Übrigen erst im Jahr 2000 entdeckt worden. Bei alledem darf aber nicht außer Acht gelassen werden, dass es ihm auch darum ging, deutsche Interessen zu verteidigen. Wenn Deutschland wieder „in den Kreis der zivilisierten Völkergemeinschaft“ gefunden hat und in der Weltpolitik viel Verantwortung übernimmt, dann ist die Forderung nach einem ständigen Sitz im UN-Sicherheitsrat - wie im März 2004 zum Ausdruck gebracht ${ }^{(12)}$ - auch legitim. Das neue und normalisierte Deutschland hat auch keinen Grund mehr, ausschließlich aus historischer Verpflichtung zu handeln.

Doch nun drängt sich die Frage auf: Wie wird das „normalisierte“ und selbstbewusste Deutschland in Frankreich wahrgenommen? Die Kommentare in der französischen Presse und der politische Diskurs in Frankreich scheinen Gerhard Schröder Recht zu geben. Tatsächlich stieß seine Anwesenheit bei den Feierlichkeiten auf keine nennenswerte Kritik, in vielen Fällen wurde sie sogar ausdrücklich positiv bewertet ${ }^{(13)}$. Die Bedeutung, die diesem historischen Ereignis beigemessen wurde, wird in

8 Siehe „Bundeskanzler Gerhard Schröder: Namensartikel in der Zeitung Ouest France“ (Anm. 4).

9 Richard von Weizsäcker hatte anlässlich des 40. Jubiläums des Kriegsendes erklärt: „Der 8. Mai war ein Tag der Befreiung. Er hat uns alle befreit von dem menschenverachtenden System der nationalsozialistischen Gewaltherrschaft." Die vollständige Rede ist auf der Internetseite des Bundespräsidialamtes verfügbar: http://www.bundespraesident.de/SharedDocs/Downloads/DE/Reden/2015/02/150202RvW-Rede-8-Mai-1985.pdf;jsessionid=68CA4CC98ED4F0972ECDBA2A30048231.2_cid293?__ blob=publicationFile (zuletzt aufgerufen am 11.04.2016). Eine Analyse dieser Rede ist zu finden in: Dirk Schmaler, Die Bundespräsidenten und die NS-Vergangenheit - zwischen Aufklärung und Verdrängung, Beiträge zur Aufarbeitung der NS-Herrschaft, Frankfurt am Main, 2013.

10 Dies trifft im Übrigen auf die französische Öffentlichkeit ebenso wie auf die deutsche zu.

11 Siehe dazu beispielsweise Marc-Olivier BEHRER, „Gerhard Schröder incarne la génération d'aprèsguerre“, La Croix, 04.06.2004 und Jacques-Pierre Gougeon, „L’Allemagne deux fois émancipée“, Le Monde, 04.06.2004.

12 Zur „Kampagne um einen Uno-Sicherheitsratssitz“, siehe Stephan Bierling, Vormacht wider Willen, München, 2014, S. 146-150.

13 Eine quantitative Untersuchung hat Elisa Eidam, zwar mit einem etwas anderen Quellenkorpus, in ihrer Untersuchung unternommen. Siehe Elisa Eıdam, Frankreich und die „Berliner Republik“, Hamburg, 2014, S. 318-322. 
einem Artikel, der am Folgetag in Libération veröffentlicht wurde, gut zum Ausdruck gebracht: „Die Verwandlung Deutschlands wurde am Sonntag zusammen mit der Befreiung Europas von den alten Gespenstern des revanchistischen Nationalismus und der totalitären und rassistischen Herrschaftsträume, die in der Vergangenheit zu Blutvergießen geführt haben, feierlich besiegelt“(14). Die deutsche Teilnahme am D-Day-Gedenken ist also für die französischen Kommentatoren die offizielle Anerkennung des „neuen Deutschlands“, von dem Gerhard Schröder sprach. In seiner Ansprache ging Jacques Chirac in die gleiche Richtung, indem er, sich an seinen Gast wendend, erklärte, dass dieser Deutschlands Erneuerung verkörpere, und dass er einer Generation angehöre, die „mit dem Willen hervortrat, ein neues Land, eine beispielhafte Demokratie in Anknüpfung an die universellen Werte der Menschenrechte und der Freiheit aufzubauen“ und Deutschland „wieder zu seinem Platz unter den europäischen Nationen und innerhalb der Gemeinschaft der Nationen verhalf“(15). Es sind keine Aussagen von hochrangigen französischen Politikern bekannt, die sich gegen die Einladung Gerhard Schröders ausgesprochen haben. Jacques Chirac ging in seiner Rede sogar noch weiter, indem er zum Schluss seinem Gast erklärte: „An diesem Tag des Gedenkens und der Hoffnung empfangen die Französinnen und Franzosen Sie mehr denn je als Freund. Sie empfangen Sie als Bruder“(16). Wer hätte im Mai 1945 gedacht, dass ein französischer Präsident dies jemals einem deutschen Staatsvertreter sagen würde? Diese Aussage macht auf eindrückliche Weise deutlich, welch langer Weg seit dem Zweiten Weltkrieg zurückgelegt wurde. Interessant ist sie in zweifacher Hinsicht. Erst einmal brachte Jacques Chirac damit zum Ausdruck, dass seiner Ansicht nach eine neue Stufe in den deutsch-französischen Beziehungen erreicht wurde: Brüder sind schließlich noch enger verbunden, als Freunde es sein können. Interessant ist darüber hinaus, dass Jacques Chirac seinen Gast im Namen des Volks empfing und damit auf die beim Besuch ausländischer Gäste übliche Begrüßung im Namen des Staats verzichtete. Nicht der Staat Frankreich empfing Gerhard Schröder als Bruder, sondern „die Französinnen und Franzosen“. Nicht jeder dürfte dem zustimmen. Immer wieder wird darauf hingewiesen, dass die deutsch-französische Freundschaft sich auf politischer und wirtschaftlicher Ebene bewährt habe, aber von Deutschen und Franzosen in ihrem alltäglichen Leben oft nicht gelebt werde. Fest steht aber, dass eine breite Mehrheit der Franzosen die Einladung Gerhard Schröders begrüßt hat. Einer im Vorfeld durchgeführten Umfrage zufolge, die in den Medien viel zitiert wurde, stieß sie bei $88 \%$ der Befragten auf Zustimmung. Gleichzeitig gaben

14 Siehe Patrick Sabatier, „Réconciliations“, Libération, 07.06.2004. Im Original heißt es: „La métamorphose de l'Allemagne a été consacrée solennellement dimanche, en même temps que la libération de l'Europe des vieux démons du nationalisme revanchard et des rêves de domination totalitaire et raciste qui l'ont ensanglantée par le passé."

15 Siehe „Rede Jacques Chiracs in Caen“, 06.06.2004, verfügbar unter : www.european-security.com/n_ index.php? id=4591 (zuletzt aufgerufen am 11.04.2016). Im Original heißt es: „Vous appartenez à cette génération née dans les ruines, qui s’est affirmée avec la volonté de construire un pays nouveau, une démocratie exemplaire, dans la fidélité aux valeurs universelles des droits de l'homme et de la liberté. Cette génération qui a su restaurer la place de l'Allemagne, foyer de l'humanisme et berceau de la pensée, parmi les nations européennes, au sein de la communauté des nations."

$16 E b d$. Im Original heißt es: „En ce jour du souvenir et de l'espérance, les Françaises et les Français vous reçoivent plus que jamais en ami. Ils vous reçoivent en frère." 
82\% der Befragten an, Deutschland als den zuverlässigsten Verbündeten Frankreichs zu betrachten ${ }^{(17)}$. Auch wenn der Irakkrieg an diesen Werten nicht ganz unschuldig sein dürfte, sind sie beachtlich. Selbst unter den Veteranen war die Ablehnung einer deutschen Teilnahme am D-Day-Gedenken nicht mehr so groß. Es erhoben sich nur einzelne Stimmen dagegen, andere dagegen begrüßten sie sogar ${ }^{(18)}$. Ein weiterer Hinweis für die veränderte Wahrnehmung Deutschlands in Frankreich liegt in der neuen Bewertung des deutschen Widerstands. 2004 wurde dieser erstmals von französischer Seite auf höchster Staatsebene gewürdigt. So verlieh Noëlle Lenoir, damals Ministerin für europäische Angelegenheiten, am 28. Januar 2004 dem früheren Wehrmachtoffizier Philipp Freiherr von Boeselager, der zu den letzten Überlebenden des Attentatsversuchs auf Hitler am 20. Juli 1944 gehörte, die Ehrenlegion ${ }^{(19)}$. Am gleichen Tag fand in Paris ein Treffen zwischen französischen und deutschen Widerständlern statt.

Auch wenn die Teilnahme Gerhard Schröders an der Gedenkfeier zum 60. Jahrestag des D-Days mit der neuen Wahrnehmung Deutschlands zusammenhängt, gibt es eine Reihe politischer, bzw. tagespolitischer Faktoren, die sie gefördert haben. Dabei handelt es sich in erster Linie um den Irakkrieg, den damaligen Stand der deutschfranzösischen Beziehungen und die Vertiefung des europäischen Integrationsprozesses. Um den Rahmen nicht zu sprengen, können diese Aspekte nur angerissen werden. Der französische Journalist Daniel Vernet vertrat in einem Gastbeitrag in der Frankfurter Rundschau am 5. Juni 2004 folgende These: „Gleichwohl sollte die Ehre, die der Geschichte erwiesen wird, nicht die Diplomatie, also die Politik, total verdrängen. Jacques Chirac ist sicher nicht unzufrieden, den deutschen Kanzler an seiner Seite zu haben, wenn er einen US-Präsidenten empfängt, der sich in einen Krieg stürzte, dem die französische und die deutsche Regierung ihre Zustimmung verweigerten “(20). Über diese Deutung ließe sich freilich streiten, aber es gibt tatsächlich eine Verbindung zwischen dem Irakkrieg und der Einladung Gerhard Schröders zum D-Day-Gedenken. Im Juni 2004 war der transatlantische Konflikt immer noch nicht überwunden und

17 Die Ergebnisse der IFOP-Umfrage wurden von Jérôme Fourquet und Magali Gérard in: Le Figaro präsentiert und erklärt: „L'Allemagne est devenue le meilleur allié de la France“, Le Figaro, 05.06.2004.

18 Lediglich einzelne Widerstandverbände äußerten sich kritisch: Über die Association Nationale des anciens combattants de la Résistance wurde gemeldet, dass sie geteilter Meinung war, da sie eine Abschwächung des Sinns des Kampfes der Alliierten gegen die Wehrmacht befürchtete (siehe „L'Allemagne est invitée aux 60 ans du Débarquement“, La Croix, 05.01.2004), und Jacques Vico, Vorsitzender des normannischen Veteranenverbands Union des combattants volontaires de la résistance du Calvados, vertrat die Meinung, dass die Deutschen ,in dieser Gedenkfeier nichts zu suchen“ hätten, da es nach der Landung „noch ein Jahr des Kriegs und des Leidens“ gegeben habe, und die Deutschen „Zivilisten getötet und bis zum Schluss Leute vertrieben“ hätten (siehe Frédéric CнамвоN, „En Normandie, l'invitation du chancelier Schröder est, pour les uns, une évidence, pour d'autres, une douleur“, Le Monde, 05.06.2004. Im Original heißt es: „Après juin 1944, il y eut encore une année de guerre et de souffrance [...]. Les vétérans allemands n’ont pas leur place dans la commémoration. Ils ont tué des civils et déporté des gens jusqu'au bout.“). Robert Chambeiron dagegen, der an der Seite von Jean Moulin eine wichtige Rolle im Widerstand eingenommen hatte, bezeichnete die Anwesenheit Gerhard Schröders in der Normandie als „gute Nachricht“ (siehe Robert Chambeiron, „La Libération sans le peuple?", L’Humanité, 04.06.2004).

19 Siehe „Rede von Noëlle Lenoir in Paris“, 28.01.2004, verfügbar unter: http://discours.vie-publique.fr/ notices/043000403.html (zuletzt aufgerufen am 11.04.2016).

20 Siehe Daniel Vernet, „Europas Ideale“, Frankfurter Rundschau, 05.06.2004. 
die gemeinsame Ablehnung des Irakkriegs durch Deutschland und Frankreich hat zu einer erheblichen Verbesserung des bilateralen Verhältnisses geführt, die die Einladung Gerhard Schröders wiederum gefördert hat. Um das zu verstehen, muss man sich daran erinnern, dass die ersten Jahre der Zusammenarbeit zwischen Jacques Chirac und Gerhard Schröder alles andere als konfliktfrei verliefen. Nach seinem Amtsantritt im Herbst 1998 machte der neue deutsche Kanzler seine Absicht deutlich, die Interessen seines Landes in der EU selbstbewusster zu verteidigen und wollte vor allem in die Zukunft schauen: Von der belasteten deutschen Vergangenheit wollte er sich emanzipieren, er wollte nicht mehr ausschließlich aus historischer Verpflichtung handeln ${ }^{(21)}$. Bezeichnend ist, dass er unmittelbar nach seiner Wahl die Einladung Jacques Chiracs zur Teilnahme am 80. Jahrestag des Waffenstillstands von 1918 ablehnte. So kam es insbesondere zu verschiedenen Konflikten auf dem Gebiet der Europapolitik, die im Dezember 2000 im Rahmen der Verhandlungen zum Vertrag von Nizza in eine Krise von zuvor selten dagewesenem Ausmaß gipfelten ${ }^{(22)}$. Zwar kam es daraufhin zu Initiativen zur Überwindung dieser Krise, doch die bilateralen Beziehungen erreichten erst 2002/2003 im Vorfeld und während des Irakkrieges eine ganz andere Qualität. Beide Länder arbeiteten zwischen dem Herbst 2002 und dem Frühjahr 2003 so eng zusammen wie noch nie zuvor ${ }^{(23)}$. Das gemeinsame „Nein“ zum Irakkrieg ermöglichte zudem den Übergang von einer rein pragmatischen zu einer sowohl auf politischer als auch auf persönlicher Ebene guten und engen Zusammenarbeit. Zwei hoch symbolische Gesten zeugen davon. Am 17. Oktober 2003 ließ sich Gerhard Schröder bei einem EU-Gipfel von Jacques Chirac vertreten, um im Bundestag an einer wichtigen Debatte teilnehmen zu können ${ }^{(24)}$. Die zweite Geste ist die Einladung Gerhard Schröders zum Gedenken an die Landung in der Normandie, die ebenfalls Ende 2003 ausgesprochen wurde. Das erklärt vielleicht, weshalb sich die Feierlichkeiten am 6. Juni 2004 in einen Festakt für die deutsch-französische Freundschaft verwandelt haben und den USA dagegen, die 1944 eigentlich die entscheidende Rolle gespielt hatten, im Vergleich wenig Beachtung geschenkt wurde. Ebenfalls von großer Bedeutung ist der europäische Integrationsprozess, der sich 2004 in einer entscheidenden Phase befand und in der Bevölkerung ebenso wie in der Politik höchst umstritten war: Ein Monat vor dem D-Day-Gedenken traten zehn neue Länder der EU bei und diese war gerade dabei, sich eine Verfassung zu geben. In diesem Kontext löste der Integrationsprozess in weiten Teilen der europäischen Bevölkerung wachsende Angst und Skepsis aus. Jacques Chirac und Gerhard Schröder wandten sich in ihrer Rede an eben diese Euroskeptiker, um sie mit dem Argument zu überzeugen, dass die Europäische Integration nicht die Laune einer Handvoll Politiker sei, sondern vielmehr eine moralische Verpflichtung gegenüber der Vergangenheit. In diesem Kontext war es also wichtiger denn je, in der europäischen Bevölkerung ein Zusammengehörigkeitsgefühl, ja,

21 Siehe dazu Wichard Woy Ke, Deutsch-französische Beziehungen seit der Wiedervereinigung. Das Tandem faßt wieder Tritt, Opladen, 2000, S. 170-171.

22 Siehe dazu ebd, S.179-181 und Gisela MüLler-Brandeck-Bocquet, Frankreichs Europapolitik, Wiesbaden, 2004, S. 207-211.

23 Siehe dazu beispielsweise G. Müller-Brandeck-Bocquet, ebd., S. 253-259.

24 Siehe Gregor Schöllgen, Die Außenpolitik der Bundesrepublik Deutschland, München, 1999, S. 243-244 
eine Art europäische Identität, zu fördern. Dem Willen, aus dem Zweiten Weltkrieg so etwas wie einen europäischen Erinnerungsort $\mathrm{zu}$ machen, stand jedoch die sehr unterschiedliche Lage der einzelnen Länder während des Kriegs im Wege: Die Einen waren Opfer der Verbrechen der Anderen; die Einen gehörten zum Lager der Sieger, die Anderen zu dem der Besiegten. Auch wenn sich die Wirklichkeit selbstverständlich nicht auf diese Schwarz/Weiß-Darstellung beschränkt - um nur ein Beispiel zu nennen: Die „Siegermacht Frankreich“ hatte selbst mit den Nationalsozialisten kollaboriert und auch in Deutschland gab es Widerständler -, konnte eine gemeinsame Erinnerung aller Länder an den Krieg nur durch eine Betonung der Gemeinsamkeiten erreicht werden, die manchen als Geschichtsklitterung erscheint. Ein Satz ganz am Anfang der Rede Jacques Chiracs ist dafür besonders beispielhaft: „Die Landung in der Normandie war das blutige Aufeinandertreffen von Tausenden Soldaten, alliierten Soldaten und deutschen Soldaten. Der 6. Juni 1944 steht aber vor allem für die Wiedererlangung der Freiheit und die Wiederherstellung der Demokratie auf dem europäischen Kontinent, der von der Ideologie des Nazismus und ihrem mörderischen Wahn unterdrückt wurde“(25). Das allmähliche Verschwinden der Unterscheidung zwischen Siegern und Besiegten, Oper- und Täterstaaten, ist die sichtbarste Erscheinungsform dieses Willens, aus dem Zweiten Weltkrieg einen europäischen Erinnerungsort mit gegenwarts- und zukunftsgerichteter Botschaft zu machen.

Im Vorfeld der Reise Gerhard Schröders in die Normandie kam es jedoch in Deutschland zu einer - wenn auch kleinen - Polemik, die teilweise auch von den französischen Kommentatoren wiedergegeben wurde. Der Kanzler entschied sich nämlich kurzfristig gegen einen Besuch des großen deutschen Soldatenfriedhofs La Cambe mit der Begründung, dass dort auch Angehörige der Waffen-SS liegen würden. Stattdessen kündigte er an, den Friedhof in Ranville besuchen zu wollen, auf dem neben Gefallenen der Commonwealth-Staaten auch 322 deutsche Soldaten begraben sind ${ }^{(26)}$. Diese Entscheidung stieß bei Teilen der Opposition auf heftige Kritik: Peter Ramsauer, Geschäftsführer der CSU-Landesgruppe im Bundestag, bezeichnete sie in der BildZeitung beispielsweise als „Beleidigung für die vielen Bundeswehrsoldaten, die diese Gräber pflegen, und für alle deutschen Kriegerwitwen “(27). Der Historiker Reinhard Rürup dagegen hielt die Entscheidung für richtig: „Der D-Day wird begangen als ein Tag der entscheidenden Wende im Kampf gegen das Dritte Reich. An einem solchen Tag kann man nicht diejenigen besonders würdigen, die das Dritte Reich verteidigt haben “(28). Diese Aussage ist überraschend, in Wirklichkeit ging es ja niemandem darum, die Anhänger des „Dritten Reichs“ zu würdigen, zumal auf dem deutschen

25 Siehe „Rede Jacques Chiracs in Caen“ (Anm. 15). Im Original heißt es: „Le débarquement de Normandie a été le face-à-face sanglant de milliers de combattants, soldats alliés et soldats allemands. Mais le 6 juin 1944 marque surtout le renouveau de la liberté et de la démocratie sur un continent européen opprimé sous le joug de l'idéologie nazie et de sa folie meurtrière.“

26 Siehe „Regierungssprecher Anda weist Kritik am Besuchsprogramm des Bundeskanzlers in der Normandie zurück“, Presse- und Informationsamt der Bundesregierung, Pressemitteilung, Nr. 279, 03.06.2004.

27 Zitiert nach „D-Day - Opposition attackiert Schröders Normandie-Programm“, Spiegel Online, 04.06.2004.

28 Zitiert nach „D-Day - Schröder ist willkommen“, Focus, 04.06.2004. 
Soldatenfriedhof La Cambe bei Weitem nicht nur Angehörige der Waffen-SS liegen. Und weil die Geschichte manchmal ironisch ist, ist viele Jahre später bekannt geworden, dass auch in Ranville Soldaten der Waffen-SS begraben sind ${ }^{(29)}$. Die Polemik erinnert jedenfalls sehr stark an die Kritik, die Helmut Kohl wegen seines Besuchs des Soldatenfriedhofs von Bitburg mit US-Präsident Ronald Reagan am 5. Mai 1985 erfahren hatte, weil dort 49 Angehörigen der Waffen-SS begraben sind ${ }^{(30)}$. Aus all dem geht die Frage hervor, ob die Tatsache, dass es 20 Jahre nach Bitburg zu einer ähnlichen Polemik kam, nicht gerade davon zeugt, dass sich Deutschland von seiner Vergangenheit in Wirklichkeit gar noch nicht emanzipiert hat, bzw. immer noch nicht unverkrampfter mit ihr umging, und dass die Nachkriegszeit im Gegensatz zu Gerhard Schröders Behauptung 2004 immer noch nicht vorbei war, dass es also immer noch keine deutsche „Normalität“ gab. Dieser Eindruck scheint sich zu bestätigen, wenn man sich die Rede Gerhard Schröders in Caen genauer anschaut. An zwei Stellen hob er nämlich die besondere Pflicht hervor, die Deutschland auf Grund seiner Geschichte seiner Meinung nach zukomme, und zwar wies er zum Einen darauf hin, dass die „Soldatenfriedhöfe und die Narben der beiden Weltkriege [...] den Völkern Europas, besonders dem deutschen Volk, eine andauernde Pflicht auferlegt [hätten]“, nämlich „Rassismus, Antisemitismus und totalitären Ideologien zu widerstehen“, und erklärte zum Anderen, dass „Europa [...] seine Lektion gelernt [habe]“ und dass „gerade [die] Deutschen [...] sie nicht verdrängen [würden]“(31). Stehen beide Aussagen nicht im Widerspruch zum Auftritt Deutschlands als normalisierte Nation? Wenn die Bundesrepublik ein Staat ist wie jeder andere auch, kann ihr dann eine besondere Pflicht zukommen? Und was ist mit dem Willen Gerhard Schröders, eben nicht mehr nur aus historischer Verpflichtung zu handeln? Oder sind die Äußerungen zur besonderen Verantwortung, bzw. Pflicht Deutschlands vielleicht nur ein Zugeständnis an die ausländischen Zuhörer?

Gerade angesichts der angesprochenen Grenzen deutscher Normalität und des besonderen Kontextes des Jahres 2004 empfiehlt es sich, zumindest einen kurzen Blick auf den nächsten großen Jahrestag im Juni 2014 zu werfen. Die Einladung Deutschlands, so kann zunächst festgestellt werden, war eine Selbstverständlichkeit. So wurde bereits im Februar 2014 vermeldet, dass der französische Präsident François Hollande Gerhard Schröders Nachfolgerin Angela Merkel zur Teilnahme an den für Juni geplanten Gedenkveranstaltungen eingeladen hat, und dass sie bereits zugesagt hat ${ }^{(32)}$. Die deutsche Beteiligung war mittlerweile sogar so selbstverständlich geworden, dass dieser Information in den deutschen und französischen Medien kaum Beachtung geschenkt wurde. Im Mittelpunkt der Medienberichterstattung im Vorfeld der Feierlichkeiten standen ebenfalls ganz andere Themen: die Frage der Teilnahme Wladimir Putins und

29 Siehe Markus Meckel, „Arbeit für den Frieden - Versöhnung über den Gräbern“, Friedrich-EbertGedächtnisvortrag, Heidelberg, 04.02.2015, verfügbar unter: https:/www.volksbund.de/fileadmin/ redaktion/BereichUeberUns/Praesident/Meldungen/2015/150204_Rede_Markus_Meckel_Friedrich_ Ebert_Gedenkstaette.pdf (zuletzt aufgerufen am 11.04.2016).

30 Siehe dazu beispielsweise Henning KöHler, Helmut Kohl - Ein Leben für die Politik. Die Biografie, Köln, 2014, S. 492-500.

31 Siehe „Rede von Bundeskanzler Gerhard Schröder“ (Anm. 3).

32 Siehe beispielsweise „Commémorations: Merkel en France le 6 juin“, Le Figaro.fr/AFP, Le Figaro Newsflash, 19.02.2014. 
die Ukraine-Krise, und in etwas geringerem Maße auch die Folgen der Europawahlen, die Ende Mai stattgefunden hatten. Genau wie ihr Vorgänger veröffentlichte Angela Merkel einen Gastbeitrag in Ouest France ${ }^{(33)}$ und entschied sich für einen Besuch des Soldatenfriedhofs in Ranville. Ist das D-Day-Gedenken 2014 also eine Wiederholung von 2004 nur mit anderen Akteuren? Nicht ganz, denn es gibt auffällige - und große - Unterschiede: Binationale Zeremonien zwischen Frankreich und den wichtigsten an der Landung beteiligten Staaten wurden auch dieses Mal organisiert, eine deutschfranzösische Gedenkfeier gab es jedoch nicht ${ }^{(34)}$. Dies hatte auch zur Folge, dass Angela Merkel der internationalen Eröffnungszeremonie lediglich als Zuschauerin beiwohnte, ohne das Wort zu ergreifen. Auch deshalb war anzunehmen, dass die Berichterstattung über ihre Anwesenheit deutlich geringer ausfallen würde als bei ihrem Vorgänger, zumal es durch das Fehlen einer deutsch-französischen Gedenkfeier auch kein symbolträchtiges Bild des deutsch-französischen Paars geben konnte. Stellt der 70. Jahrestag des D-Day unter diesem Gesichtspunkt also einen Rückschritt dar im Vergleich zum 60.? Man kann zunächst nicht außer Acht lassen, dass der Kontext im Juni 2014 bei Weitem nicht so günstig war wie zehn Jahre zuvor. Die Christdemokratin Angela Merkel und der Sozialist François Hollande hatten, nicht zuletzt auf Grund der wirtschaftlichen Lage ihres jeweiligen Landes, aber auch aus parteipolitischen Gründen, sehr unterschiedliche Vorstellungen davon, wie auf die 2009 ausgebrochene Eurokrise zu reagieren sei ${ }^{(35)}$. Das deutsch-französische Paar war in keiner so guten Verfassung wie 2004. Es ist unmöglich, das auszublenden, wenn man sich die Frage stellt, warum Deutschland bei der Ausgestaltung der Feierlichkeiten keinen größeren Platz eingeräumt wurde. Es auf diesen Aspekt zu reduzieren wäre aber zu kurz gegriffen. Man sollte nicht vergessen, dass es auch 2004 maßgeblich auf den besonderen Kontext zurückzuführen war, dass das D-Day-Gedenken zu einem Fest der deutsch-französischen Freundschaft geworden ist, und es ist immerhin zur Selbstverständlichkeit geworden, dass Deutschland an den Jubiläumsfeierlichkeiten beteiligt wird. Außerdem bestätigt eine Äußerung François Hollandes in seiner Festrede vor allen ausländischen Staats- und Regierungschefs, dass Deutschland nicht mehr als reiner Täterstaat wahrgenommen wird: „Ich möchte den Mut der Deutschen würdigen, die auch Opfer des Nazismus waren und in einen Krieg hineingezogen wurden, der nicht der ihre war und der nicht der ihre hätte sein sollen “(36). Interessant ist, dass Deutschland und seine Kanzlerin auf Grund des internationalen Kontextes bei der Gedenkveranstaltung auf

33 Siehe Angela Merkel, „Tag der Befreiung und der Dankbarkeit“, Ouest France, 06.06.2014.

34 Informationen zum Programm sind zu finden in einer Pressemappe des französischen Präsidialamtes. Siehe Dossier de presse - Présidence de la République, „Commémoration du $70^{\mathrm{e}}$ anniversaire de la libération de la France“, Normandie - Vendredi 6 juin 2014, verfügbar unter : www.elysee.fr/assets/ Uploads/6juin1944.pdf (Stand: 12.02.2016).

35 Siehe dazu beispielsweise Pierre-Emmanuel Thomann, „Le couple franco-allemand et la dimension géopolitique de la crise de l'euro“, Hérodote, 4 (2013), n 151, S. 39-59.

36 Siehe „Rede François Hollandes bei der internationalen Gedenkveranstaltung zum 70. Jahrestag der Landung in der Normandie“, Ouistreham, 06.06.2014, verfügbar unter: http:/www.elysee.fr/ declarations/article/ceremonie-internationale-de-commemoration-du-70e-anniversaire-dudebarquement-en-normandie-ouistreham-dday7/ (Stand: 16.10.2015). Übersetzung von www.zeit.de/ news/2014-06/06/konflikte-wiedersehen-mit-putin-bei-feiern-zum-d-day-in-normandie-06064602. 
eine andere Art und Weise im Mittelpunkt standen: Frankreich wollte letztere nutzen, um dafür zu sorgen, dass der Dialog zwischen Russland und der Ukraine wieder aufgenommen wird; bei den Gesprächen spielte Angela Merkel eine entscheidende Rolle ${ }^{(37)}$. Ist nicht gerade das ein Zeichen dafür, dass es so etwas wie eine „deutsche Normalität“ gibt? Die Berliner Republik tritt selbstbewusst auf, übernimmt in der Weltpolitik Verantwortung, wird von ihren internationalen Partnern zunehmend losgelöst von der belasteten Vergangenheit wahrgenommen, befindet sich mit ihnen auf Augenhöhe, und erinnert sich zusammen mit ihnen an die Verbrechen der Vergangenheit. Ob es auch bedeutet, dass die Nachkriegszeit zu Ende ist, sei dahingestellt.

\section{Quellen- und Literaturverzeichnis}

\section{Quellen}

„Rede von Noëlle Lenoir in Paris“, 28.01.2004, verfügbar unter: http://discours.vie-publique.fr/ notices/043000403.html (zuletzt aufgerufen am 11.04.2016).

„Regierungssprecher Anda weist Kritik am Besuchsprogramm des Bundeskanzlers in der Normandie zurück, Presse- und Informationsamt der Bundesregierung“, Pressemitteilung, Nr. 279, 03.06.2004.

Gerhard SchröDER, „Ces morts, nous les avons tous en commun“, Le Figaro, 05.06.2004.

„Bundeskanzler Gerhard Schröder: Namensartikel in der Zeitung Ouest France“, Presse- und Informationsamt der Bundesregierung, 05.06.2004.

„Rede von Jacques Chirac in Caen“, 06.06.2004, verfügbar unter: www.european-security. com/n_index.php? id=4591 (zuletzt aufgerufen am 11.04.2016).

„Rede von Bundeskanzler Gerhard Schröder bei den französisch-deutschen Feierlichkeiten des „D-Day' am 6. Juni 2004 in Caen“, Bulletin der Bundesregierung, Nr. 58-4 vom 06.06.2004.

Gerhard SCHRöDER, Entscheidungen - Mein Leben in der Politik, Hamburg, 2006.

Angela Merkel, „Tag der Befreiung und der Dankbarkeit“, Ouest France, 06.06.2014.

Dossier de presse - Présidence de la République, „Commémoration du $70^{\mathrm{e}}$ anniversaire de la libération de la France, Normandie“ - Vendredi 6 juin 2014, verfügbar unter: www.elysee.fr/ assets/Uploads/6juin1944.pdf (zuletzt aufgerufen am 11.04.2016).

„Rede von François Hollande bei der internationalen Gedenkveranstaltung zum 70. Jahrestag der Landung in der Normandie“, Ouistreham, 06.06.2014, verfügbar unter: www.elysee.fr/ declarations/article/ceremonie-internationale-de-commemoration-du-70e-anniversairedu-debarquement-en-normandie-ouistreham-dday7/ (zuletzt aufgerufen am 11.04.2016).

Unterschiedliche Artikel aus den Zeitungen Le Figaro, Le Monde, Libération, La Croix und L'Humanité.

„D-Day - Opposition attackiert Schröders Normandie-Programm“, Spiegel Online, 04.06.2004.

„D-Day - Schröder ist willkommen“, Focus, 04.06.2004.

\section{Literatur}

Christine Axer, Die Aufarbeitung des NS-Vergangenheit - Deutschland und Österreich im Vergleich und im Spiegel der französischen Öffentlichkeit, Köln/Weimar/Wien, 2011.

Stephan BIERLING, Vormacht wider Willen - Deutsche Außenpolitik von der Wiedervereinigung bis zur Gegenwart, München, 2014.

Gisela Müller-Brandeck-Bocquet, Frankreichs Europapolitik, Wiesbaden, 2004.

37 Siehe dazu bsp. Isabelle Lasserre, „Moscou et Kiev renouent le dialogue en Normandie“, Le Figaro, 07.06.2004 oder „Hollande diplomate: mission réussie“, Le Monde, 08.06.2014. 
Elisa EIdAM, Frankreich und die „Berliner Republik“ - Wandel nationaler Identität und politische Neuorientierung im vereinigten Deutschland aus Sicht der französischen Presse, Hamburg, 2014.

Marcus Hawel, Die normalisierte Nation - Vergangenheitsbewältigung und Außenpolitik in Deutschland, Berlin, 1998.

Henning KöHLer, Helmut Kohl - Ein Leben für die Politik. Die Biografie, Köln, 2014.

Markus Meckel, Arbeit für den Frieden - Versöhnung über den Gräbern, Friedrich-EbertGedächtnisvortrag, Heidelberg, 04.02.2015, verfügbar unter: https://www.volksbund.de/file admin/redaktion/BereichUeberUns/Praesident/Meldungen/2015/150204_Rede_Markus_ Meckel_Friedrich_Ebert_Gedenkstaette.pdf (zuletzt aufgerufen am 11.04.2016).

Gerhard Schröder, Entscheidungen - Mein Leben in der Politik, Hamburg, 2006.

Pierre-Emmanuel Тномаn,, „Le couple franco-allemand et la dimension géopolitique de la crise de l'euro", Hérodote, 4 (2013), n 151, S. 39-59.

Wichard Woyke, Deutsch-französische Beziehungen seit der Wiedervereinigung - Das Tandem fasst wieder Tritt, Wiesbaden, 2004.

\title{
Résumé
}

Le 6 juin 2004, en la personne de Gerhard Schröder, un chancelier allemand a participé pour la première fois aux cérémonies commémoratives du débarquement allié en Normandie. Le fait que l'Allemagne ait été invitée à commémorer avec les Alliés le début de la fin de la domination nazie sur l'Europe est le signe que quelque chose a changé. Ce geste hautement symbolique témoigne-t-il de la fin définitive de la période d'après-guerre, comme l'a indiqué Gerhard Schröder en amont des festivités ? Est-ce l'illustration de la "normalité» retrouvée de l'Allemagne? Comment cette dernière se présente-t-elle en France soixante ans après la fin de la Seconde Guerre mondiale et quinze ans après la chute du Mur de Berlin et comment y est-elle perçue ? L'objectif de cet article est de tenter de répondre à ces questions.

\begin{abstract}
On june the 6th 2004, in the person of Gerhard Schröder, a German Chancellor participated for the first time in the commemorative ceremonies of the Allied landings in Normandy. The fact that Germany has been invited to commemorate the beginning of the end of Nazi domination of Europe with the Allies is a sign that something has changed. Is this highly symbolic gesture showing that the post-war period is definitively finished, as stated by Gerhard Schröder in the runup to the festivities? Is it the illustration of Germany's recovered "normality"? How the Federal Republic is representing itself in France sixty years after the end of World War II and fifteen years after the fall of the Berlin Wall and how is it perceived there? The purposes of this article are to attempt to answer these questions.
\end{abstract}

